\title{
Le témoignage des camps et sa médiation préfacielle
}

The Camps Testimony and its Prefatory Mediation

\section{Fransiska Louwagie}

\section{OpenEdition}

1 Journals

Édition électronique

URL : http://journals.openedition.org/questionsdecommunication/7724

DOI : 10.4000/questionsdecommunication. 7724

ISSN : 2259-8901

Éditeur

Presses universitaires de Lorraine

Édition imprimée

Date de publication : 1 décembre 2006

Pagination : 349-367

ISBN : 978-2-86480-828-2

ISSN : 1633-5961

Référence électronique

Fransiska Louwagie, "Le témoignage des camps et sa médiation préfacielle », Questions de communication [En ligne], 10 | 2006, mis en ligne le 01 décembre 2006, consulté le 22 mars 2021. URL : http://journals.openedition.org/questionsdecommunication/7724; DOI : https://doi.org/ 10.4000/questionsdecommunication.7724 


\title{
$>$ NOTES DE RECHERCHE
}

\author{
FRANSISKA LOUWAGIE \\ Fonds de la recherche scientifique, Flandre \\ Katholieke Universiteit Leuven \\ Fransiska.Louwagie@kuleuven-kortrijk.be
}

\section{LE TÉMOIGNAGE DES CAMPS ET SA MÉDIATION PRÉFACIELLE}

Résumé. - Cet article vise à développer un modèle pour l'analyse de la préface du témoignage portant sur l'expérience concentrationnaire. La préface constitue un canal de médiation entre le lecteur et le texte : elle remplit à la fois un rôle promotionnel et une fonction de traduction interculturelle orientée vers le publiccible. À partir du modèle préfaciel proposé par Gérard Genette, de certaines théories de l'analyse du discours, ainsi que des analyses récentes du paratexte postcolonial par Richard Watts, nous élaborons une grille de lecture s'appliquant aux types de médiation conçus par les différentes catégories de préfaciers.

Mots clés. - Médiation, témoignage des camps de concentration, préface allographe, préface autographe, traduction interculturelle, analyse du discours. 
$\mathrm{D}$ epuis 60 ans, les témoignages écrits des camps de concentration se sont accumulés. Ils manifestent une grande hétérogénéité, qui se reflète d'ailleurs dans les appellations génériques utilisées à leur sujet: « document », « récit », « autobiographie »... En effet, plusieurs démarches testimoniales sont envisageables, allant de la simple attestation des faits au texte narratif élaboré au plan littéraire. À quoi s'ajoute que les témoins ont souvent ressenti le besoin de légitimer leur propre démarche dans une préface, ou de faire intervenir une autorité externe susceptible de soutenir l'approche en question!. Dans le présent article, nous proposons d'étudier ces préfaces, et ce dans une double perspective : l'analyse de la médiation du texte testimonial par la préface et celle de la particularisation de la préface dans le domaine testimonial. Nous mettrons plus précisément à l'épreuve les théories préfacielles, avant d'élaborer une grille d'analyse. Précisons encore qu'il s'agira principalement, dans ce qui suit, de la préface « allographe », c'est-à-dire du cas de figure où le préfacier ne coïncide pas avec l'auteur du texte. Cela étant, nous fournirons en complément quelques dispositifs d'analyse adaptés à l'étude de la préface « autographe » ou préface d'auteur. Pour des raisons que nous aurons l'occasion d'expliciter plus loin, notre corpus sera limité à des exemples francophones.

\section{La préface allographe}

À qui incombe-t-il d'assurer le passage de l'intime au public au moyen d'une préface? À première vue, l'hétérogénéité des démarches testimoniales correspond à la diversité des préfaciers sollicités, car ceuxci proviennent d'horizons relativement variés. Néanmoins, il y a lieu de les ranger en quatre catégories majeures. Les premières sont celles du champ littéraire et du champ historiographique, deux genres de discours auxquels le témoignage est habituellement associé (Louwagie, 2003 : 366). Dans le domaine littéraire, on retrouve des écrivains comme François Mauriac (1947:9-12²; $1957: 9-12 ; 1958$ : 9-14), Georges Duhamel (1948: 7-8) ou Louis Aragon (1954:7-14). Par exemple, François Mauriac a rédigé des préfaces pour trois témoignages différents, dont La Nuit, texte célèbre d'Elie Wiesel. Le domaine historique est représenté en particulier par Pierre Vidal-Naquet (1992a : i-ix ; 1996 : I 1-22). Nous disposons de deux préfaces écrites de sa main pour des

\footnotetext{
' La préface tient son rôle médiateur du fait qu'elle appartient à la « zone intermédiaire entre le hors-texte et le texte » (Compagnon, 1979 : 328). Elle constitue, d'une part, un lieu de passage pour le lecteur (Compagnon, 1979 : 328 ; Rigolot, 1987 : 7) et, d'autre part, elle est l'un des éléments paratextuels les plus tournés vers le texte même (Couturier, $1995: 48$ ).

${ }^{2}$ La préface figure aussi dans des rééditions (Mauriac, I965: II-13; 1985 : |3-14).
} 
témoignages francophones ${ }^{3}$. La troisième scène d'énonciation est celle des témoins historiques canonisés. Dans le domaine francophone, nous pouvons citer l'exemple d'Elie Wiesel (1997 5-7 ; 2004 :5-6), qui a rédigé au moins deux préfaces pour des auteurs d'origine juive écrivant en français. Enfin, il nous a paru indispensable d'inclure les préfaciers de la deuxième génération des survivants. Rappelons Serge Klarsfeld (1996:7-9 ; 1997 :9$\left.12^{4} ; 2002: 7-14 ; 2003: 9-11^{5}\right)$, fils de déporté et combattant pour la mémoire juive du génocide, et auteur de plusieurs préfaces de récits de survivants des camps.

Il va de soi que les différents domaines dans lesquels les préfaciers sont ancrés vont, dans une certaine mesure, influer leurs préfaces respectives. Dans ce qui suit, il s'agira dès lors de proposer un modèle d'analyse apte à prendre en compte à la fois les facteurs généraux ou récurrents des préfaces et de montrer au sein de ceux-ci les amorces favorables à un contenu plus individualisé.

\section{Les fondements d'une théorie préfacielle : Gérard Genette}

Rappelons que Gérard Genette a attribué deux fonctions majeures à la préface allographe originale 6 . La première consiste à recommander la lecture du texte, moyennant une personnalité connue qui prête son nom et sa plume à la promotion du récit. La simple intervention de cette autorité incite à la lecture, pour ainsi dire indépendamment du texte de la préface (Genette, 1987 : 247). La deuxième fonction que Gérard Genette (1987:249)7 reconnait est celle d'orienter l'interprétation et l'appréciation du public. Nous étudierons ces deux fonctions préfacielles, tout en tenant compte d'une critique récente formulée par Richard

\footnotetext{
${ }^{3}$ Outre que des préfaces destinées à des témoignages en traduction, dont la préface au témoignage de $\mathrm{S}$. Laks. Ce témoignage avait d'abord paru en français, préfacé par $\mathrm{G}$. Duhamel (1948: 7-8), et ensuite à Londres en polonais ; la dernière version a été retraduite en français et préfacée par P. Vidal-Naquet (1992b :9-18).

${ }^{4}$ Avec B. Klarsfeld.

${ }^{5}$ Avec B. Klarsfeld.

${ }^{6}$ Les autres fonctions que G. Genette attribue à la préface allographe s'appliquent plutôt aux préfaces ultérieures.

7 En fait, G. Genette parle de la « dimension critique » de la préface. II distingue cette fonction en principe du « guidage » qu'on retrouve dans les préfaces autographes, « l'auteur étant le principal et, à vrai dire, le seul intéressé à une bonne lecture (Genette, 1987 : 183). Cependant, dans la mesure où le préfacier allographe n'est pas seulement celui qui « commente » mais aussi celui qui « expose » (J.-L. Borges, cité dans Genette, 1987 : 248), nous opterons pour une séparation moins rigoureuse entre les fonctions de la préface allographe et la préface autographe. D'ailleurs, R. Watts paraphrase la deuxième fonction de la préface comme « explanation and guidance » (Watts, 2005 : 19).
} 
Watts dans son étude du paratexte de la littérature francophone postcoloniale : il y reproche à la théorie de Gérard Genette son caractère universalisant et anhistorique. Afin de permettre une étude historiographique de la préface, le modèle nécessite, selon lui, une réinterprétation dans une perspective « culturaliste et diachronique » (Watts, 2005 : 76). Nous partageons cet « amendement » de la théorie genettienne, ainsi que le principe sous-jacent : étudier non seulement les propos que le préfacier tient sur le texte mais aussi la façon dont il noue ce dernier aux contextes culturels qui ont présidé à sa production et réception. Dès lors, notre cadre de référence méthodologique se composera de deux approches susceptibles d'articuler le rapport entre texte et contexte. D'abord, il s'agit des théories de l'énonciation développées par Dominique Maingueneau et par Ruth Amossy ; elles conviennent particulièrement bien à l'étude de la première fonction préfacielle, soit la promotion du récit. Les notions clés de ces théories seront adaptées en fonction de l'étude spécifique du paratexte testimonial. La deuxième approche est constituée des dispositifs d'analyse proposés par Richard Watts : ceux-ci serviront de point d'appui à l'étude de la deuxième fonction préfacielle, guider le lecteur.

\section{Pour une analyse discursive de la première fonction préfacielle}

Comme l'a affirmé Dominique Maingueneau (1983), l'œuvre se constitue en constituant son contexte. Sa théorie de l'analyse discursive, prolongée notamment par Ruth Amossy (2000), pose dès lors que c'est en tant que « partie du message » qu'il convient de traiter les « abords immédiats » (Maingueneau, 1993 : 23) du texte. À l'aide des outils analytiques proposés par ces deux auteurs, nous comptons examiner les positions énonciatives construites au sein de la préface. Cependant, là où l'analyse du discours porte habituellement sur le rapport entre l'énonciateur et le co-énonciateur lecteur, nous aurons - dans le cadre de la préface - à distinguer une troisième position. Celle-ci est occupée par l'auteur-témoin, qui est parfois apostrophé directement ou, de façon dialogique, construit comme un second coénonciateur. Nous devrons donc simultanément étudier la façon dont le préfacier se positionne vis-à-vis du public, la façon dont il présente l'auteurtémoin ainsi que la façon dont il se rapporte lui-même à ce dernier.

En premier lieu, il importe d'examiner les questions de la légitimité et de l'identité des locuteurs, moyennant une analyse de l'ethos prédiscursif et discursif du préfacier et de l'auteur-témoin. L'ethos se définit, au sein de l'analyse du discours, comme la « représentation » que le public est amené à

\footnotetext{
${ }^{8}$ Nous nous fonderons en particulier sur R. Amossy (2000) et D. Maingueneau (2004).
} 
construire du locuteur, ainsi que l'« adhésion 》 - ou le manque d'adhésion engendrée par cette représentation vis-à-vis des points de vue du locuteur en question. L'ethos prédiscursif est l'ethos dont le locuteur est doté avant même d'avoir pris la parole, tandis que l'ethos discursif est celui qui se construit au moment du discours (Maingueneau, 2004 :205). Sur le plan prédiscursif,il s'agit en l'occurrence de définir les différentes « scènes d'énonciation 》 auxquelles appartiennent les locuteurs. En effet, chacun se situe au sein d'un certain type de discours (ibid. : | 191 ), qui co-détermine la façon de présenter le témoignage. Comme nous l'avons annoncé, la préface testimoniale est fondée sur quatre scènes d'énonciation principales, nommément les champs littéraire et historiographique, le domaine du témoignage public et celui de la mémoire (transgénérationnelle). À cet égard, les auteurs de préfaces multiples, comme ceux mentionnés plus haut, constituent des cas particulièrement révélateurs. En effet, la comparaison des préfaces successives d'une même personne permet de reconstruire en détail l'ethos de celle-ci. Pour ce qui est de l'ethos prédiscursif accordé par le préfacier à l'auteur-témoin, la question est de savoir si cet auteur possède déjà une notoriété dans le domaine testimonial ou ailleurs : songeons par exemple à Simone Lagrange, témoin-clé du procès Barbie, ou à Walter Spitzer, qui portait témoignage à travers ses tableaux et ses sculptures, avant de passer à l'écriture.

Sur le plan discursif - explicite aussi bien qu'implicite -, le préfacier est en situation d'apporter éventuellement des modifications aux ethos respectifs. II s'agit notamment de voir comment le statut de témoin est évalué par rapport à celui d'autres « spécialistes 》 de l'histoire des camps : la posture du témoin est-elle présentée comme allant de soi ? Le témoin se voit-il doté d'un ethos d'« expert »? Existe-t-il un rapport d'infériorité ou de supériorité avec d'autres « experts » du génocide, tels que des historiens, des philosophes et des écrivains ? En même temps, le préfacier définit ses propres rapports avec l'auteur-témoin. D'un côté, il s'agit de retracer les jugements de valeur que le préfacier porte sur l'auteur et, par extension, sur le récit de celui-ci et sur le témoignage en général. De l'autre, il convient d'examiner la situation d'énonciation mise en scène par la préface. Pensons par exemple au cas de la lettre-préface, où l'énonciation intime du discours épistolaire est incorporée à l'énonciation préfacielle qui possède un caractère public ${ }^{9}$ : l'auteur-témoin y

\footnotetext{
${ }^{9}$ Nous proposerions d'étudier ce genre de conflit énonciatif à l'aide des concepts de « scène générique » et de « scénographie », développés par D. Maingueneau (2004 : 191-192). La scène générique ou genre s'inscrit dans une scène d'énonciation englobante ou type de discours (voir supra) et peut donner lieu à différentes scénographies ou « scènes narratives », qui sont construites au niveau du texte. Ainsi, dans notre exemple, la scène générique préfacielle est-elle combinée avec une scénographie épistolaire. Selon D. Maingueneau (2004 : 192), la scénographie constitue un « piège » pour le lecteur, puisque ce dernier « reçoit le texte d'abord à travers sa scénographie, non à travers sa scène englobante et sa scène générique, reléguées au second plan mais qui constituent en fait le cadre de cette énonciation ». Comme nous l'indiquons ici, l'analyse de ce « piège » nous parait notamment intéressante en cas d'écart entre la scène générique et la scénographie attendue.
} 
figure comme « destinataire-relais » (Genette, 1987: 180) pour le lectorat. Finalement, l'analyse de l'ethos discursif porte aussi sur l'image que le préfacier donne à voir de l'auditoire : est-ce que le préfacier s'adresse de préférence à un public particulier, excluant une partie du lectorat $^{10}$ ? Ces différentes perspectives d'étude vont de pair avec un examen des actes de langage qu'on pourrait appeler promotionnels : le geste de parrainage prend-il la forme d'une passation de la parole ? ; le préfacier se montre-t-il prêt à partager son propre public avec le témoinauteur ? ; le préfacier adopte-t-il une attitude normative vis-à-vis du public, moyennant des invitations ou des impératifs, et ainsi de suite" ?

Pour terminer, il convient de revenir sur le fait que l'analyse énonciative proposée par Ruth Amossy et Dominique Maingueneau implique la prise en compte du co-énonciateur lecteur (Maingueneau, 2004 : 190-191) et donc du public-cible (Amossy, 2000 : 39). La caractérisation - éventuellement implicite - du public co-conditionne incontestablement la posture énonciative du préfacier et sera également importante pour l'analyse de la deuxième fonction préfacielle. Dans ce qui suit, il s'agira notamment d'identifier le profil culturel du lectorat. Celui-ci peut faire l'objet d'une caractérisation culturelle spécifique, ou bien être considéré comme potentiellement universel, cas rare, puisque certains traits sociohistoriques demeurent souvent visibles en filigrane (Amossy, $2000: 55$ ).

\section{Pour une analyse interculturelle de la deuxième fonction préfacielle}

\section{La traduction interculturelle}

Si Gérard Genette attribue au préfacier la fonction d'offrir quelques points de repère pour une « bonne » lecture du texte, Richard Watts avance que cette deuxième mission prend, dans le contexte francophone qui est le sien, la forme d'une médiation interculturelle ou « traduction ». $\mathrm{Vu}$ les différences culturelles entre l'écrivain postcolonial et le centre culturel dominant qui l'accueille (Watts, 2005 : 76), le préfacier se voit, en d'autres termes, obligé d'assurer un processus de contextualisation. Cette conception de la traduction n'est pas nouvelle ; elle a même acquis droit de cité en traductologie, grâce entre autres travaux, à l'excellent recueil The Translatability of Cultures. Figurations of the Space

\footnotetext{
${ }^{10}$ Cette question, et notamment l'aspect culturel de la caractérisation du public, réapparaîtra au sein de la deuxième fonction préfacielle.

"Les fonctions connative et expressive du discours préfaciel allographe sont discutées dans l'excellent article de G. Idt (1977: $67 ; 72-73)$.
} 
Between édité en 1996 par Sanford Buddick et Wolfgang Iser. D'après ce dernier auteur, le concept de traduction permet de décrire l'interpénétration de différentes cultures sans lui (pré)imposer une structure organisatrice rigide (Iser, $1996: 295$ ). En même temps, Wolfgang Iser (1996: 295) étend la notion d'interculturalité : celle-ci couvre non seulement les rapports entre différentes cultures, mais aussi les rapports « intraculturels » entre passé et présent. De ce point de vue, le témoignage concentrationnaire se situe à son tour dans un processus interculturel, étant donné qu'il traite d'un environnement étranger au vécu de la majorité des lecteurs. De ce fait, nous aurons à notre tour recours au trope de la traduction pour analyser la façon dont le préfacier « interprète » les rapports entre la culture-source, en l'occurrence celle des camps, d'où témoigne le survivant, et la culturecible des lecteurs. Afin de pouvoir étudier de manière efficace les rapports interculturels, nous avons, comme on l'a dit, limité la culturecible au domaine testimonial francophone ${ }^{12}$. Force est de constater que les préfaciers renvoient à cette culture-cible au cours de leurs préfaces. Ainsi Serge Klarsfeld fait-il référence à la responsabilité du régime de Vichy, et Pierre Vidal-Naquet à la guerre d'Algérie ou aux négationnistes français.

Afin de conceptualiser le trope de la traduction interculturelle, Richard Watts emprunte et métaphorise certains concepts traductifs d'Antoine Berman. Pareille démarche n'est pas sans susciter des réserves. D'abord, Richard Watts opte pour un interlocuteur dont les théories font figure à part au sein des études de traduction. Pour les besoins de notre analyse, le caractère idiosyncrasique et peu systématique de l'apparat bermanien ne pose cependant pas de problèmes majeurs, dans la mesure où ce dispositif d'analyse partage le mécanisme binaire constitutif des autres théories traductives, notamment l'opposition entre le « même », défini à partir de la culture-cible, et l'《 autre », associé à la « culture-source 》 (voir Lane-Mercier, 1997 : 43-68 ; Buddick, 1996 : I-3). Deuxièmement, s'il s'avère que les métaphorisations de Richard Watts ouvrent des perspectives d'étude intéressantes, les transitions opérées sont parfois trop hâtives pour être entièrement convaincantes. Par exemple, rappelons que Richard Watts (2005: 76) ambitionnait d'intégrer les concepts genettiens dans un cadre « culturaliste ». Or, Barbara Godard (200I : 64) 13 a montré que, si la théorie d'Antoine Berman aborde la traduction comme une donnée culturelle, elle n'a pas pour objet la

\footnotetext{
${ }^{12}$ Celui-ci couvre les auteurs et les préfaciers français, avec une prise en compte de certaines interactions franco-belges et de la culture juive française ou francophone.

${ }^{13}$ Le caractère essentialiste de la théorie d'A. Berman a également été relevé par A. Brisset (1998: 4I), qui montre d'ailleurs la contradiction entre les «présupposés essentialistes » d'A. Berman et certaines de ses affirmations d'inspiration « historicofonctionnaliste ».
} 
culture en tant qu'élément sociohistorique, mais « en tant que rayonnement de l'esprit », l'approche d'Antoine Berman étant essentialiste plutôt que fonctionnaliste. Afin de doter les concepts bermaniens des visées analytiques sollicitées par Richard Watts, il est donc impératif de les intégrer à une conception sociohistorique de la notion de culture. Finalement, Richard Watts définit les concepts empruntés à Antoine Berman (1985 :26-27) de manière peu rigoureuse : d'une part, il fonde son interprétation des termes sur un seul passage, sans tenir compte des différents points de vue, parfois plus détaillés, parfois contradictoires, qu'Antoine Berman à émis ${ }^{14}$; d'autre part, Richard Watts n'explicite pas toujours les significations nouvelles que les concepts traductifs reçoivent au sein de l'étude paratextuelle. Dans ce qui suit, nous allons présenter les concepts utilisés par Antoine Berman et par Richard Watts, en procédant à une sélection et à une redéfinition critique de certains d'entre eux.

\section{Un système binaire}

Dans le passage cité par Richard Watts, Antoine Berman oppose deux types de traduction - l'une appelée « ethnocentrique »; l'autre « éthique »- qu'il caractérise à l'aide d'une double dichotomie complémentaire, reprise dans la grille ci-dessous :

\begin{tabular}{ll} 
ethnocentrique & éthique \\
\hline hypertextuelle & poétique \\
\hline platonicienne & pensante
\end{tabular}

Antoine Berman situe chaque dichotomie à un niveau différent: l'opposition entre « ethnocentrique » et « éthique » est d'ordre culturel, celle entre « hypertextuel » et « poétique » d'ordre littéraire et celle entre « platonicien » et « pensant » d'ordre philosophique. Les définitions qu'offre Antoine Berman des différents termes restent cependant très sommaires, voire lacunaires. En ce qui concerne la première dichotomie, on peut comprendre que la pratique « ethnocentrique » se caractérise par le fait qu'elle subordonne les valeurs du texte aux valeurs de la culture de réception (Berman, 1985 : 29), et la pratique « éthique » par son respect pour la différence culturelle du texte-source. Ensuite, l'hypertextualité constitue, en traduction, « un travail de reformulation » (ibid. : 38), fondé sur un « lien d'engendrement libre » (ibid. : 36). L'approche poétique implique alors une plus grande fidélité au texte-source. Nous n'avons pas retrouvé d'analyse des attitudes «pensante » et « platonicienne »

\footnotetext{
${ }^{14}$ À propos des contradictions dans l'œuvre d'A. Berman, nous renvoyons à l'article de B. Godard (200I : 63-7I)
} 
dans le passage d'Antoine Berman auquel renvoie Richard Watts. L'attitude « platonicienne » est définie dans un texte de 1986 et, en résumé, elle signifie « ce qui dissocie le sens de la forme et ne cherche qu'à traduire le sens » (Berman, 1986 : 63-64).

Richard Watts converge avec Antoine Berman sur la signification de la première et de la troisième dichotomie ${ }^{15}$. En revanche, en ce qui concerne la deuxième dichotomie, leurs interprétations sont nettement différentes. Richard Watts reprend les termes « hypertextuel » et « poétique » mais en dotant ceux-ci d'une signification foncièrement nouvelle. En effet, au fil de ses analyses, il redéfinit l'approche poétique comme une « relation horizontale avec le texte » (Watts, 2005 : 83). Cette posture, qui met le préfacier sur un pied d'égalité avec l'auteur et son texte, et la posture verticale opposée, c'est-à-dire l'attitude supérieure du préfacier vis-à-vis du texte, se situent sur le plan de l'évaluation (littéraire) du texte, plutôt que sur celui de l'analyse des rapports traductifs. Ainsi cette dichotomie recoupe-t-elle les jugements de valeur que nous avons distingués à l'intérieur de la première fonction préfacielle. Or, Richard Watts ne se contente pas de modifier l'acception des termes bermaniens. Simultanément, il propose une « nouvelle » dichotomie qui, en dernière analyse, semble précisément couvrir le même domaine d'application que l'opposition proposée initialement par Antoine Berman. Richard Watts (2005 : 133) oppose les préfaciers qui

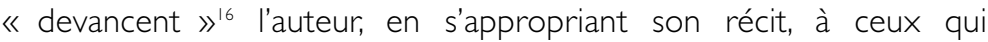
« doublent » l'auteur ou lui font « écho » (Watts, 2005 : 20). Comme celle d'Antoine Berman, cette dichotomie porte vraisemblablement sur le lien de fidélité ou de liberté entre texte-source et texte-cible. Laquelle des oppositions serait alors la plus apte à décrire ce lien ? À vrai dire, aucune des notions proposées ne convient parfaitement à décrire le rapport en question, du moins dans le cadre du discours préfaciel. L'hypertexte étant défini par Gérard Genette (1982: 12) comme un texte dérivé « par une opération transformative », comme l'imitation, mais qui ne commente pas, nous jugeons en effet que ce terme peut s'appliquer à la traduction proprement dite ${ }^{17}$, mais qu'il est quelque peu réducteur pour la préface, puisque celle-ci, si elle peut recourir à certains mécanismes d'imitation, tient aussi du « commentaire $»^{18}$. L'on a donc affaire à une relation de « second degré » dont les dispositifs méritent d'être analysés en d'autres termes que le « second degré » de

\footnotetext{
${ }^{15}$ Nous précisons que le terme « pensant » est traduit par « reflexive » dans le texte de R. Watts.

${ }^{16}$ Notre traduction ; le terme anglais utilisé par R. Watts est « precede ».

${ }^{17}$ Avant A. Berman, G. Genette (1982 :238-243) a d'ailleurs opéré lui-même le lien entre hypertexte et traduction.

${ }^{18}$ La notion de « commentaire » est associée par G. Genette (1982 : 9-10) au métatexte, mais également au paratexte.
} 
I'hypertexte (ibid. : 12) ${ }^{19}$. Les termes choisis par Richard Watts ne sont pas plus appropriés. De fait, celui d' « écho » risque de prêter à confusion, étant donné qu'il a servi, chez Dan Sperber et Deirdre Wilson (198| : 556-559), à définir un rapport intertextuel de «mention » (ironique), par opposition à un rapport d'《 usage ». Pour corroborer l'écart entre une relation de commentaire ou d'imitation, et celle d'une mention (ironisante), nous proposons de remplacer le terme d'« écho » par celui d'《 embrayage », au sens où Antoine Compagnon (1979 :4243) entend ce dernier dans son expression « embrayage à friction $»^{20}$. L'embrayage y implique qu'un texte transmet son mouvement à un autre, engendrant un « travail de citation »-rappelons à ce propos que le terme de « citation » reçoit chez Antoine Compagnon (ibid. : 32) une interprétation large et inclut également les rapports de commentaire. Par opposition au concept d'embrayage, nous pouvons qualifier de « débrayage » l'interruption du mouvement de friction avec le texte-source : le préfacier n'entre pas dans un rapport d'imitation stylistique avec le texte ou impose ses propres idées au récit. Dans le dernier cas, il risque d'annexer ou d'instrumentaliser le « sens », afin de défendre ce que Gérard Genette (1987 : 250) a appelé, précisément dans le contexte de la préface allographe, une « cause plus vaste, ou éventuellement toute différente ». Au lieu que la préface soit un pré-

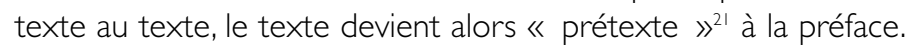

\section{Un système interactif}

Selon le passage bermanien auquel se réfère Richard Watts, les rapports entre les différentes attitudes traductives sont fixés de manière très stricte. Ainsi la traduction ethnocentrique est-elle « nécessairement » hypertextuelle, en plus d'être « l'envers exact » de la traduction éthique et de ses corrélés respectifs (Berman, 1985 :27, 30). En revanche, chez Richard Watts les parallélismes et les oppositions n'acquièrent pas de caractère absolu. Ses analyses montrent en effet que les préfaces combinent plusieurs approches traductives (Watts, 2005 : 20, 83). Comme quoi, il devient possible, si l'on accepte d'envisager les concepts d'Antoine Berman comme potentiellement interactifs, de prendre en

\footnotetext{
${ }^{19} \mathrm{G}$. Genette utilise l'expression « second degré » non seulement pour l'hypertexte, mais aussi pour le métatexte ou « commentaire ».

${ }^{20}$ || s'agit d'un embrayage de « second degré », différent de l'embrayage discursif sur la situation d'énonciation décrit par É. Benveniste (1966:238-245 et $1974: 80-88$ ) ; les deux modalités d'embrayage ne s'excluent pas et se côtoient d'ailleurs à l'intérieur de la préface.

${ }^{21}$ « L'œuvre préfacée devient alors simple prétexte à un manifeste, à une confidence, à un règlement de compte, à une divagation » (Genette, 1987 :250).
} 
compte la complexité des préfaces, y compris leurs éventuels paradoxes internes. Comment donc recombiner les mêmes termes dans un réseau moins contraignant ? Premièrement, nous estimons que la dichotomie de départ n'est pas celle entre l'approche ethnocentrique et l'approche éthique, mais celle entre la traduction platonicienne et la traduction pensante. Ensuite, il semble bien que les autres approches traductives peuvent se manifester sur chacun de ces deux plans.

Commençons par observer que, dans l'article de 1986 voué à la traduction platonicienne, Antoine Berman (1986: 63-64) définit les activités traductives à partir de la double composante saussurienne du signe : le « sens » et la « lettre ». L'activité de traduction peut se situer à chacun de ces deux niveaux ${ }^{22}$. La traduction du « sens » correspond à l'approche platonicienne et veut respecter et articuler la « logique signifiée » du texte. La traduction de la « lettre », qu'on pourrait dire « littérale » plutôt que « pensante », tend au contraire à transmettre le travail sur la langue. Cependant, elle vise plus loin que le simple « calque », car elle veut traduire la « logique de la lettre »(Berman, 1985:|4|). D'après Antoine Berman (ibid. : 88, |4I), elle réalise, à cette fin, un travail sur la langue-source autant que sur la langue-cible, permettant de découvrir à toutes les deux de nouvelles couches linguistiques. Or, cette affirmation ouvre la voie à un bouleversement du modèle dichotomique initial. Le travail sur la propre langue montre, en premier lieu, que l'approche ethnocentrique ne va pas, d'office, de pair avec l'optique platonicienne : l'ethnocentrisme peut se manifester tant au niveau du « signifié » qu'à celui du « signifiant », tout comme l'approche éthique est susceptible de se déployer sur les deux plans. En ce qui concerne la logique « signifiée », il y va alors de la reconnaissance de différences culturelles et des rapports d'opposition ou de hiérarchie entre ces cultures : l'écart culturel est-il souligné ou minimisé ? La question à retenir au plan du « signifiant » porte sur les rapports entre la logique langagière du témoin et celle du public : est-ce que l'idiolecte et la logique « littérale » du témoin écrivant en français sont considérés comme différents de ceux du lecteur francophone, et si oui, pour quelles raisons ? S'agit-il seulement d'un décalage historique entre production et réception du texte ou y a-t-il un indicible ou un problème communicationnel dû à l'expérience « extrême »? Selon le préfacier, les tensions langagières nous apprennent-elles quelques choses sur la culture-source et/ou sur la culture-cible ? Deuxièmement, il s'avère que les attitudes ethnocentrique et éthique ne s'excluent pas, puisque la traduction peut prendre en compte simultanément la culture-source et

\footnotetext{
${ }^{22}$ Selon A. Berman (1986:64), signifié et signifiant forment une unité dans tout texte original, tandis que la traduction instaure une « expérience de la langue » où les deux sont dissociés.
} 
la culture-cible. À la dialectique de la Bildung allemande, propreétranger-propre, Antoine Berman (1985: 86) oppose lui-même la théorie de Friedrich Hölderlin, où « l'épreuve de l'étranger» et « l'apprentissage du propre » ont lieu simultanément et se tiennent en équilibre. Afin de traiter ces deux aspects d'une manière neutre, nous remplacerons le concept normatif d'« éthique » par celui d'« allocentrique ».

Les autres dichotomies peuvent également relever des composantes « signifiée » ou « signifiante » du texte. C'est d'abord le cas de l'opposition entre embrayage et débrayage. Pour ce qui est du signifié, le préfacier peut entrer en rapport de dialogue avec les conceptions de l'auteur-témoin, ou au contraire imposer ses idées préconçues au récit. Au niveau stylistique, un mécanisme analogue peut être identifié lorsque le préfacier « imite » le style de l'auteur. C'est le cas où la préface entre dans un rapport proprement dit « hypertextuel » avec le texte. Relativement à la littérature postcoloniale, Richard Watts (2005:20) décrit l'exemple de la préface qui imite la créolisation du texte. De même, dans notre corpus, le préfacier peut reprendre l'idiolecte de l'auteur-témoin, que celui-ci soit « concentrationnaire », religieux ou autre.

En dernier lieu, signalons que l'opposition entre l'attitude horizontale et l'attitude verticale du préfacier, que nous rattachons - à l'opposé de Richard Watts - à la première fonction préfacielle, est à son tour susceptible de se déployer sur les plans du « sens » et de la «lettre », suivant que l'admiration ou le dénigrement préfacier vont vers le contenu ou l'élaboration du récit en question. II s'agit là d'une relation souvent inversement proportionnelle, étant donné que Richard Watts (2005: I I, I43, I 46) ${ }^{23}$ rattache l'attitude horizontale à la reconnaissance de l'autonomie littéraire du récit alors que l'attitude verticale est définie comme la soumission du texte à des valeurs extralittéraires. Si le préfacier affirme donc la pertinence du récit pour un domaine du savoir non littéraire, son attitude, horizontale au niveau du « signifié », risque d'être perçue comme verticale au niveau du « signifiant », si la préface passe outre aux caractéristiques formelles du texte.

\footnotetext{
${ }^{23}$ Sur la question de l'autonomie littéraire, l'approche du préfacier risque en fait de revêtir des significations contradictoires: l'inclusion dans le domaine littéraire peut être envisagée comme signe de respect ou comme une forme d'annexion culturelle; de même, la reconnaissance d'un domaine testimonial peut signifier une exclusion du domaine littéraire ou, au contraire, intégrer une valorisation positive des textes.
} 


\section{Grille d'analyse}

Sur la base de ce qui précède, nous pouvons résumer les différents axes en les intégrant à une grille de lecture qui s'applique aux deux fonctions préfacielles, c'est-à-dire la promotion du récit, d'une part, et le guidage du lecteur par un processus de traduction interculturelle, de l'autre :

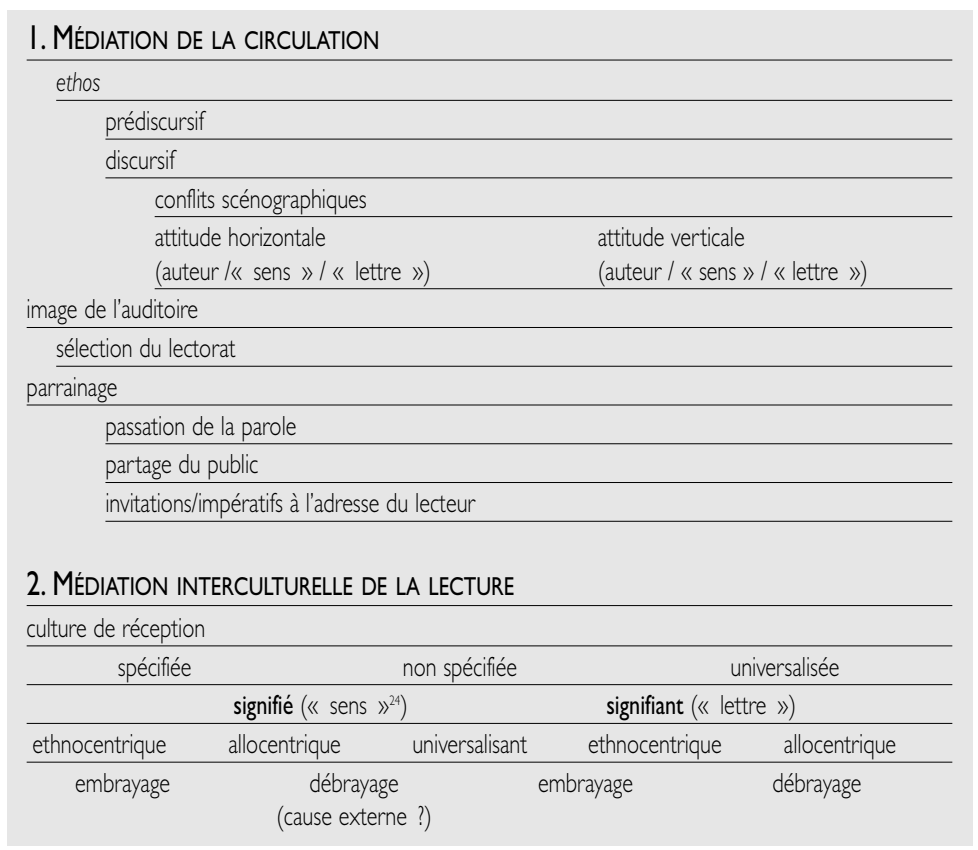

\section{La préface autographe}

Dans la préface autographe, la première fonction médiatrice, celle qui consiste à inciter à la lecture, se présente évidemment sous un autre angle que dans la préface allographe « parrainée ». Comme l'a montré Gérard Genette (1987 : 184), l'auteur-préfacier s'interroge en priorité sur le «pourquoi » de l'écriture et de la lecture, en s'excusant du « comment », c'est-à-dire de sa façon (insuffisante) de traiter le sujet. En effet, une telle posture est probable dans le cas du témoignage, où l'on sait qu'il est usuel, y compris dans la préface, d'insister sur l'importance éthique du sujet et sur le caractère non littéraire de son propre récit. Cette double prise de position, éthique et a-littéraire, inclut

\footnotetext{
${ }^{24}$ Dans notre modèle d'analyse, ces plans transcendent les différentes dichotomies, là où, chez A. Berman, l'optique (non) platonicienne constituait une attitude traductive supplémentaire. Nous jugeons le recours à ce terme philosophique peu précis et, dans notre interprétation, superflu.
} 
potentiellement la revendication d'un ethos d'expert de la part du témoin, comparable à celui évoqué dans le contexte de la préface allographe : l'ethos en question repose, d'une part, sur la volonté de discréditer d'autres interlocuteurs spécialisés dans le sujet des camps (Trépos, 1996 :23), tels les écrivains et les historiens, et d'autre part sur une « rhétorique du besoin », qui souligne l'utilité des témoignages pour la société (ibid. : 57). Dans le cas d'une prise de distance à l'endroit de la scène littéraire, la façon dont le préfacier-auteur représente son propre acte d'écriture est éclairante. Si l'écriture est présentée comme hâtive et impulsive (Biron, 2003 : 60-64), et qu'elle se déroule dans des circonstances précaires, sur un « vieux cahier », parfois tenus sur les genoux, faute de table (Maous, 1996 :23), le témoin aspire en effet à un ethos de spontanéité, en contraste avec celui de l'écrivain professionnel, qui met plutôt l'accent sur l'effort et la régularité de l'écriture, comme l'ont montré les analyses de Nathalie Heinich (2000: 24). L'ethos professionnel de l'expert testimonial s'accompagne alors d'un ethos d'auteur non professionnel. Cela dit, à titre plus exceptionnel, certains témoins ne manqueront pas de revendiquer une identité d'« écrivain » et se montreront soucieux ou conscients de la composition du récit ${ }^{25}$. Enfin, chacune à leur façon, les deux catégories sont susceptibles d'évoquer la nécessité « existentielle » de l'acte d'écriture (ibid. : I |8). L'ethos de l'auteur demande dès lors aussi une mise en rapport avec certaines questions identitaires ${ }^{26}$.

Pour bien guider la lecture du récit, soit la deuxième fonction de la préface, les témoins ont souvent tendance à détailler leurs intentions d'écriture. L'hommage aux morts et la transmission de la mémoire aux vivants sont évidemment les motifs les plus fréquents. Cependant, les témoins peuvent également placer ou recontextualiser leur témoignage dans le cadre d'une cause plus large, par exemple politique ou idéologique, ou au contraire tenter de décontextualiser leur expérience, pour en montrer la validité transhistorique. Du fait qu'elles aspirent à dépasser l'expérience historique proprement dite, les re- et décontextualisations vont souvent de pair avec une redéfinition du contrat de lecture testimonial, entrainant parfois une « protestation de fictivité »(Genette, 1987 : 200). Dans ce cas, l'attention analytique portera en particulier sur la question de ce que Gérard Genette a appelé les « clés » de lecture : le préfacier peut revendiquer ou réfuter une lecture à clé, que celle-ci soit d'ordre historique ou autobiographique.

\footnotetext{
${ }^{25}$ Cette posture peut se rencontrer dans des préfaces originales aussi bien que dans des préfaces ultérieures.

${ }^{26}$ Nous ne revenons pas sur la sélection du public, qui se déroule de manière analogue à celle dans la préface allographe.
} 


\section{Conclusion}

L'analyse des dispositifs discursifs caractéristiques de la préface de témoignage a révélé un processus de médiation stratifié, si bien qu'il s'agissait de produire un modèle de lecture à même d'identifier l'ethos du préfacier et les approches traductives qu'il prône. Les résultats des lectures effectuées d'après ces paramètres auront intérêt à être comparés avec d'autres études de cas, de manière à faire ressortir les différences et les similarités. Une telle opportunité s'offre par excellence lorsqu'une même personne a préfacé plusieurs textes ou lorsqu'un texte à été préfacé par plusieurs personnes ou à plusieurs époques. Dans le premier cas, la comparaison permettrait par exemple d'examiner dans quelle mesure un certain préfacier manifeste une tendance répétée à se concentrer sur le « sens 》 ou la « lettre », à opter pour une traduction ethnocentrique et ainsi de suite. Ces tendances donneraient finalement des indices plus généraux sur les procédés d'embrayage ou de débrayage mis en œuvre par un préfacier : est-ce que le préfacier s'adapte aux problématiques et à « l'aspectualité » (Schaeffer, 1999 : 228), soit la perspective, de chaque texte individuel ? Ou fait-il au contraire prévaloir les mêmes enjeux dans chaque préface? Souvent, cette opposition entre adaptation et non-adaptation ne sera pas « discrète » mais, chez les différents préfaciers, une disposition dominante pourra néanmoins être décelée.

L'identification à la perspective du récit, cas de figure de l'embrayage, constitue un exercice cognitif, une « expérience de pensée » (Murzilli, 200 I). D'après Jean-Marie Schaeffer (2002) ${ }^{27}$ et Nancy Murzilli (ibid.), cet exercice cognitif est particulièrement efficace lors de la lecture de récits « contrefactuels ». Ceux-ci ouvrent « l'espace des possibles», et ils mettent en question notre conception « cristallisée » du monde, en la confrontant à la «perspective »du texte (Schaeffer, 2002)28. Par

\footnotetext{
${ }^{27}$ À partir de prémisses différentes, N. Murzilli et J.-M. Schaeffer arrivent à cette même conclusion.

${ }^{28}$ Le lien qu'établit J.-M. Schaeffer entre « le monde fictionnel » et « l'espace des possibles » n'implique pas une équivalence absolue entre les deux concepts. En effet, selon une analyse antérieure de J.-M. Schaeffer, le premier ne peut être réduit au second: il ne connaît pas les mêmes « contraintes logiques », est « incomplet », c'est-à-dire non exhaustif dans ses descriptions, et aussi souvent « non homogène », parce qu'il comprend les points de vue de différents agents, des points de vue pluriels (Schaeffer, 1999 : 205-207). N. Murzilli s'oppose également à une identification entre monde fictionnel et monde possible. La différence entre les deux concepts montre le caractère non totalitaire de la fiction; de ce fait, si l'effet cognitif suscité par le récit « contrefactuel » (non propagandiste) est, grâce à l'aspectualité, « contraint » ou ciblé, il n'est pas pour autant « déterminé » (l'opposition entre « contraindre » et « déterminer » est empruntée à C. Duteil-Mougel (2004).
} 
extension, nous pouvons dire que l'effet cognitif est le plus net dans les textes qui présentent une rupture avec le monde et avec les pensées du lectorat; on sait qu'il en va ainsi dans la littérature testimoniale concentrationnaire. Cet effet sera d'autant plus considérable au sein d'un processus de médiation allocentrique.

Pour ce qui est du second cas, où les mêmes enjeux reviennent de préface en préface, il convient d'examiner si les enjeux du préfacier sont (partiellement) compatibles avec - ou antithétiques à - ceux du témoignage, ce qui permettra de vérifier une nouvelle fois si le texte sert ou non de « pré-texte » à la préface. En effet, on l'a dit, les préfaciers risquent de ne pas (toujours) respecter l'aspectualité du texte ou de procéder à une lecture sélective, en fonction de leur propre « cause ». Deux types de cause s'offrent au préfacier, qui seront donc, selon le cas, plus ou moins en accord avec les enjeux défendus par l'auteur-témoin. D'abord, la cause peut être proche du génocide et d'ordre mémoriel ou identitaire, portant par exemple sur la particularité juive de la Shoah. D'autre part, la cause en jeu peut dépasser la question du génocide et relever d'un plan idéologique plus large, par exemple religieux ou politique. De façon analogue à la re- ou décontextualisation de l'expérience dans les préfaces autographes, les éléments interprétés comme référentiels sont alors liés à un deuxième plan, atemporel ou transhistorique, pour mieux soutenir la « thèse » du préfacier.

Finalement, une opportunité de comparaison s'offre également lorsqu'un texte fait l'objet de plusieurs préfaces simultanées ou successives. Par exemple, lors de rééditions, le paratexte original peut être partiellement récusé ou au contraire complété par de nouveaux éléments, qui le soutiennent ou le contredisent. Les différentes couches de ces palimpsestes seront particulièrement révélatrices des tensions éthologiques et interculturelles à travers le temps. Dans certains cas, les changements ôtent d'ailleurs (une partie de) l'autorité présumée de la préface ou du préfacier. De même que l'analyse des tensions dichotomiques intratextuelles, la mise à nu des contradictions entre les différents paratextes d'un même témoignage confirme dès lors une hypothèse récemment formulée par Georg Stanitzek. Selon celle-ci, l'étude du paratexte finit, en dépit de ses débuts structuralistes - qui restent d'ailleurs fondamentaux - par emprunter une « direction déconstructionniste »(Stanitzek, $2005:$ 34) 29: le paratexte ne peut fonctionner pleinement qu'à condition de ne pas analyser son fonctionnement.

\footnotetext{
${ }^{29}$ Notre traduction de l'anglais « deconstructive direction ». D'ailleurs, G. Stanitzek qualifie, pour sa part, l'approche de G. Genette de « positiviste » et « micrologique », plutôt que de structuraliste. Selon G. Stanitzek (2005 : 34-35), G. Genette évite de questionner le statut de sa recherche.
} 
Le témoignage des camps et sa médiation préfacielle

\section{Références}

Amossy R., 2000, L'argumentation dans le discours. Discours politique, littérature d'idées, fiction. Comment peut-on agir sur un public en orientant ses façons de voir, de penser ?, Paris, Nathan.

Aragon L., 1954, « Préface », pp. 7-14, in : Daix P., La dernière forteresse, Paris, Les Éditeurs français réunis.

Benveniste É., 1966, Problèmes de linguistique générale, Paris, Gallimard.

— 1974, Problèmes de linguistique générale 2, Paris, Gallimard, 1980.

Berman A., 1985, La traduction et la lettre ou l'auberge du lointain, Paris, Éd. Le Seuil, 1999.

—1986, « L'essence platonicienne de la traduction », Revue d'esthétique, nouvelle série, 12, pp. 63-74.

Biron M., 2003, « L'écrivain liminaire », pp. 57-70, in : Bertrand J.-P., Gauvin L., dirs, Littératures mineures en langue majeure, Québec/Wallonie-Bruxelles, Montréal/Bruxelles, Presses de l'université de Montréal/P. Lang.

Brisset A., 1998, « L'identité culturelle de la traduction. En réponse à Antoine Berman », Palimpsestes, I I, pp. 31-5I.

Budick S., 1996, "Crises of Alterity: Cultural Untranslatability and the Experience of Secondary Otherness », pp. I-22, in : Budick S., IserW., eds., The Translatability of Cultures. Figurations of the Space Between, Stanford University Press, Stanford.

Budick S., Iser W., eds, 1996, The Translatability of Cultures. Figurations of the Space Between, Stanford University Press, Stanford.

Compagnon A., 1979, La seconde main, ou le travail de la citation, Paris, Éd. Le Seuil.

Couturier M., 1995, La figure de l'auteur, Paris, Éd. Le Seuil.

Duhamel G., 1948, « [Préface] », pp. 7-8, in : Laks S., Musiques d'un autre monde, Paris, Mercure de France.

Duteil-Mougel C., 2004 [pour la publication en ligne], « Référence et textualité : le point de vue de la sémantique interprétative », http://www.revuetexto.net/Reperes/Themes/Duteil/Duteil_Reference.html.

Genette G., 1982, Palimpsestes. La littérature au second degré, Paris, Éd. Le Seuil.

— 1987, Seuils, Paris, Éd. Le Seuil.

Godard B., 200 I, « L'éthique du traduire : Antoine Berman et le "virage éthique" en traduction $\gg, T T R, 14: 2$, pp. 49-82.

Heinich N., 2000, Être écrivain. Création et identité, Paris, Éd. La Découverte.

Idt G., 1977, «Fonction rituelle du métalangage dans les préfaces "hétérographes" », Littérature, 27, pp. 65-74.

Iser W., 1996, « Coda to the discussion », pp. 294-302, in : Budick S., Iser W., eds, The Translatability of Cultures. Figurations of the Space Between, Stanford University Press, Stanford. 
Klarsfeld B., Klarsfeld S., 1997, « Préface », pp. 9-12, in : Chitelman P., Ceux et Celles qui m'ont fait « homme ». Chronique de mes rencontres, Eyrein, R. Garry.

- 2003, «Préface », pp. 9-11, in : Chytelman P., Le courage d'espérer, Eyrein, R. Garry.

Klarsfeld S., 1996, « Préface », pp.7-9, in : Gourand J., Les cendres mêlées. Récit, Paris, Éd. Le Cherche midi.

- 2002, « Préface », pp.7-14, in :Vaisman S., Parmi les cris, un chant s'élève... Le témoignage exceptionnel d'une femme médecin déportée à Auschwitz, Paris, M. Lafon.

Lane-Mercier G., 1997, «Translating the Untranslatable: The Translator's Aesthetic, Ideological and Political Responsibility », Target, 9 : I, pp. 43-68.

Louwagie Fr., 2003, « "Une poche interne plus grande que le tout" : pour une approche générique du témoignage des camps », Questions de communication, 4, pp. 365-379.

Maingueneau D., 1993, Le contexte de l'œuvre littéraire. Énonciation, écrivain, société, Paris, Dunod.

— 2004, Le discours littéraire. Paratopie et scène d'énonciation, Paris, A. Colin.

Maous F., 1996, Coma. Auschwitz, nº A.5553, Préface de Pierre Vidal-Naquet, Genève, Éd. Le Comptoir.

Mauriac F., 1947, « Préface », pp. 9-12, in : Halkin L. E., À l'ombre de la mort, Paris/Tournai, Casterman.

— 1957, « Préface », pp. 9-12, in : Maurel M., Un camp très ordinaire, Paris, Éd. de Minuit.

— 1958, « Préface », pp. 9-14, in :Wiesel E., La Nuit, Paris, Éd. de Minuit.

— 1965, « Préface », pp. I I-13, in : Halkin L. E., À l’ombre de la mort, Gembloux, Éd. Duculot.

— 1985, «Préface », pp. 13-14, in : Halkin L. E., À l'ombre de la mort, Paris/Gembloux, Éd. Duculot.

Murzilli N., 200I, «La fiction ou l'expérimentation des possibles», http://www.fabula.org/effet/interventions/I I.php.

Rigolot F., 1987, « Prolégomènes à une étude du statut de l'appareil liminaire des textes littéraires 》, L'esprit créateur, $27: 3$, pp. 7- 8 .

Schaeffer J.-M., 1999, Pourquoi la fiction ?, Paris, Éd. Le Seuil.

— 2002, « De l'imagination à la fiction », http://www.vox-poetica.org/t/fiction.htm.

Sperber D.,Wilson D., 1981, « Irony and the Use-Mention Distinction », pp. 550563, in : Davis S., ed., 1991, Pragmatics : A reader, Oxford, Oxford University press, (article publié originellement pp. 295-318, in : Cole P., ed., 1981, Radical Pragmatics, New York, Academic Press).

Stanitzek G., 2005, « Texts and Paratexts in Media », Critical Inquiry, 32, pp. 27-42.

Vidal-Naquet P., 1992a, « Préface. Une bien belle école... », pp. i-ix, in : Heftler N., Si tu t’en sors... Auschwitz, 1944-1945, Paris, Éd. La Découverte. 
Le témoignage des camps et sa médiation préfacielle

- 1992b, « Préface. Les harmoniques de Simon Laks », pp. 9-18, in : Laks S., Mélodies d'Auschwitz, Paris, Éd. du Cerf.

— 1996, « Préface.Tâche de tenir... », pp. II-22, in : Maous F., Coma.Auschwitz, $n^{\circ}$ A.5553, Genève, Éd. Le Comptoir.

Watts R., 2005, Packaging Post/Coloniality . The Manufacture of Literary Identity in the Francophone World, Lanham/Boulder/New York/Toronto/Oxford, Lexington books.

Wiesel E., 1997, « Préface », pp. 5-7, in : Lagrange S., Coupable d'être née. Adolescente à Auschwitz, Paris, Éd. L'Harmattan.

- 2004, « Préface », pp. 5-6, in : Spitzer W., Sauvé par le dessin, Paris/Lausanne, Favre. 